\title{
Cryopreserved Homografts in Infected Infrainguinal Fields are Associated with Frequent Reinterventions and Poor Amputation Free Survival
}

S. Keisin Wang MD, Ashley R. Gutwein MD, Natalie A. Drucker MD, Michael P. Murphy MD, Andres Fajardo MD, Michael C. Dalsing MD, Raghu L. Motaganahalli MD, and Gary W. Lemmon MD

\author{
Indiana University School of Medicine \\ Department of Surgery \\ Division of Vascular Surgery \\ Indianapolis, IN
}

\section{Corresponding Author:}

S. Keisin Wang MD

Integrated Vascular Surgery Research Fellow

Indiana University School of Medicine

Department of Surgery

Division of Vascular Surgery

1801 N. Senate Blvd MPC2-3500

Indianapolis, IN 46202

Phone: 317-962-0282

Fax: 317-962-0289

Email: wangkei@iupui.edu

Keywords: cryopreserved homografts, critical limb ischemia, infection, conduit

\section{Presentation:}

The project outlined in this manuscript was submitted as an abstract for oral presentation to VESS 2018 (Vail, Colorado).

This is the author's manuscript of the article published in final edited form as:

Wang, S. K., Gutwein, A. R., Drucker, N. A., Murphy, M. P., Fajardo, A., Dalsing, M. C., ... Lemmon, G. W. (2018). Cryopreserved Homografts in Infected Infrainguinal Fields Are Associated with Frequent Reinterventions and Poor Amputation-Free Survival. Annals of Vascular Surgery. https://doi.org/10.1016/j.avsg.2017.10.032 
31

32

33

\section{Abstract}

Objective:

Single-length saphenous vein continues to be the conduit of choice in infected-field critical limb ischemia (CLI). However, half of these individuals have inadequate vein secondary to previous use or chronic venous disease. We reviewed our outcomes of infected-field infrainguinal bypasses performed with cryopreserved homografts $(\mathrm{CH})$, a widely-accepted alternative to autogenous vein in this setting.

\section{Methods:}

This is a retrospective, institutional descriptive analysis of infected-field infrainguinal revascularizations between 2012-2015.

\section{Results:}

Twenty-four operations were performed in the same number of patients for limb ischemia with signs of active infection. The mean age of the cohort examined was $62.5 \pm 14.4$ (standard deviation) years. Mean SVS risk score was 3.9 with a baseline Rutherford's chronic ischemia score of 4.3 at presentation. Emergent procedures constituted 29\% of cases and the remainder were urgent. The $\mathrm{CH}$ bypass captured was a reoperative procedure in all but one of the patients. Culture positivity was present in $75 \%$ of cases with $S$. aureus (29\%) the most commonly isolated organism.

30-day mortality and major adverse cardiovascular events were both 4\%. Amputation free survival (AFS) was 75\% at 30-days. Similarly, 30-day reintervention was 38\% with debridement (43\%) and bleeding (29\%) the most common indications. 
Average duration of follow-up was $27.9 \pm 20.4$ months (range $0.5-60.4$ ). Mean length of

57 stay was 14.8 days. Reinfection requiring an additional procedure or antibiotic regimen separate

58 from the index antibiotic course was 13\%. Primary patency and AFS at 1-year was 50\% and 58\%,

59 respectively. Primary patency and AFS at 2-years was 38\% and 52\%, respectively. Limb salvage at

60 one and 2 -years was $70 \%$ and $65 \%$. Fifteen (63\%) patients required reintervention during the

61 follow-up period with $40 \%$ of those subjects undergoing multiple procedures.

62

63 Conclusions:

64

CHs remain a marginal salvage conduit in the setting of infection and no autogenous

65 choices. Therefore, clinicians should individualize usage of this high-cost product in highly selected 66 patients only. 
67

Introduction

68

69

70

71

72

73

75

76

Autogenous single-length vein continues to remain the gold standard conduit for lower extremity infected-field revascularizations for critical limb ischemia (CLI). ${ }^{1}$ However, up to $45 \%$ of patients who require bypass do not have adequate continuous vein secondary to chronic venous disease or previous vein harvest.2,3 Unfortunately, the use of alternative synthetic conduits such as PTFE (polytetrafluroethylene) puts the patient at increased risk for graft infection and limb loss.

74 Therefore, cryopreserved autologous homografts (CHs) have become a popular alternative in the 75 infected surgical field. The purpose of this retrospective analysis was to define outcomes for 6 contemporary use of $\mathrm{CHs}$ in infected fields with respect to patency and limb salvage. 

2

\section{Methods}

After obtaining Indiana University Institutional Review Board (IRB) approval, a singlecenter retrospective review was completed of all infected-field CHs implanted for infrainguinal arterial disease from 2012 to 2015 . All procedures were performed at one institution by a group of nine academic surgeons. Patients not seen by a vascular surgeon in our system for 12 -months were deemed lost to follow-up; for those, contact by phone was attempted.

Demographics captured included age, sex, disease severity, and relevant comorbidities. Presence of infection was defined as observation of cellulitis overlying a bypass graft or perigraft purulence/fluid/air on imaging. Operative characteristics captured included location of proximal/distal anastomosis, muscle flap usage, and intraoperative cultures. Post-operative management strategies queried included use of anticoagulation, antiplatelets, and antibiotics duration. CHs implanted were kept on-site in a liquid nitrogen dewar. These conduits were prepared per manufacturer's instruction but not routinely seromatched to the host. Based on availability and surgeon preference, the choice of cryopreserved vein or artery was made on a case-by-case basis.

CHs were used as the first choice in infected fields during this time over rifampin soaked prosthetic and spliced autogenous vein if continuous vein was not available. All infected fields were copiously irrigated with antibiotic and saline solution. No antibiotic impregnated beads were implanted in our series.

After surgery, all patients maintained IV or PO antibiotic use depending on the clinical severity of infection. In general, it was our practice to extend antibiotics to 4-weeks before a 
102

103

104

105

106

107

108

109

110

111

112

113

114

decision on additional duration was made in the outpatient setting. Post-operative imaging, vascular labs, and overall management was left to the discretion of the individual attending surgeon. Most commonly, a post-operative wound check was scheduled two to four weeks after the index procedure. Graft surveillance was scheduled for every three months for the first year followed by every six months thereafter. After the second year of follow-up, patients were extended to annual visits if the bypass remained patent.

Events captured included one and 2-year primary patency by vascular labs or CTA, one and 2-year amputation free survival (AFS), reinterventions, reinfection, anastomotic bleeding, mortality, and major adverse cardiovascular events (MACE). AFS was defined as freedom from allcause mortality or above-ankle amputation. Reinfection was defined as any decline in clinical status secondary to a new or persistent infection resulting in escalation of antibiotics, drainage, or reoperation to revise the index bypass graft. 


\section{Results}

From 2012 to 2015, 24 infrainguinal CH (33\% vein) bypasses were performed secondary to an infected conduit $(n=23)$ or native artery $(n=1)$. The mean age of our population was $62.5 \pm 14.4$ years (Table 1). The most common comorbidities included previous bypass (96\%), HTN (92\%), active smoking (58\%), HLD (54\%), CAD (42\%), and DM (25\%).

\section{Indications}

Twelve percent of the patients were referred acutely after initial evaluation by an outside vascular surgeon for definitive management. Mean Rutherford's chronic limb ischemia score and mean SVS risk score were 4.3 and 3.9, respectively.,5 Emergent procedures (performed within 6 hours of admission) constituted 29\% of cases and the remainder were urgent (within 24 hours). All patients demonstrated signs of local infection on physical exam or imaging; however, only $8 \%$ were septic at the time of presentation. All but one of the procedures were performed as a repeat bypass. This exception was a male with a primary infection of the superficial femoral artery secondary to chronic IV drug use and accidental arterial injection.

\section{Intra-operative}

Most of the infections were located in the groin (66.7\%). All proximal sites of anastomosis were distal to the external iliac artery. Distal targets were divided into tibioperoneal (17\%), belowknee (4\%), and above-knee (79\%) categories. Three patients received an extranatomic bypass consisting of two obturator bypasses and a femoral to femoral bypass via a retrorectus tunnel. Upon exploration, $33 \%$ of the patients had a pseudoaneurysm at the presumed site of infection 
140

141

142

\section{2}

(Table 2). The majority (92\%) of the infected conduits were unincorporated into the soft tissue.

Frank purulence was noted in $46 \%$ of limbs. Complete graft explantation was completed in $61 \%$, and the remainder received a partial explant at the location of active infection. Rotational muscle flaps were utilized in $46 \%$ of cases. Seventy-five percent of cultures returned an identifiable organism (Table 3). The most common isolated organisms were S. aureus (29\%), P. aeruginosa (24\%), and coagulase negative Staphylococcus (24\%). Eighteen percent of positive cultures further demonstrated extended spectrum antibiotic resistance.

\section{Post-operative and 30-day Outcomes}

After the index operation, $38 \%$ of patients received therapeutic anticoagulation while $88 \%$ received antiplatelet therapy (Table 4). All subjects received either IV (92\%) or PO antibiotics in the peri-operative period. The average duration of antibiotic coverage after surgery was 4.6 weeks. 30-day AFS was $75 \%$ with a mortality rate of $4 \%$. The lone death occurred in an individual presenting with peri-graft fluid and sepsis. Antibiotic sensitive S. aureus was isolated from cultures, but the patient continued to decline clinically resulting in multi-system organ failure and eventual withdrawal of care by the family. One patient experienced stroke/MI, suffering from an NSTEMI several days post-operatively. Three (13\%) patients experienced anastomotic bleeding with two requiring takebacks for exploration. Reintervention at 30-days was $38 \%(n=7)$ most commonly for further debridement (4/7) or bleeding (2/7). Average length of stay was 14.8 days.

\section{Overall Outcomes}

Mean follow-up for our population was $27.9 \pm 20.4$ months (Table 5). Primary patency in our population at one and 2-years was 50\% and 38\%. AFS at one and 2-years was 58\% (6 
amputations, 5 deaths) and 50\% (7 amputations, 7 deaths). Limb salvage at one and 2-years was

$16670 \%$ and $65 \%$, respectively. Reintervention rate during follow-up was $63 \%$ with $40 \%$ of these

167 patients requiring repeat bypass. The most common cause of reintervention was for stenosis or

168 occlusion; one third of reinterventions were for debridement or drainage. There were no

169 additional episodes of anastomotic bleeding during long-term follow-up compared to the three

170 observed within 30-days. Thirteen percent of patients had reinfection of the implanted CH. Seven

171 patients (29\%) required major amputation (3 BKA, 4 AKA) during follow-up. More than half, 54\%

172 of all treated patients died during the follow-up period (Figure 1). Of these 11 deaths, 4 were from

173 unknown causes outside of our hospital system. The remainder of deaths occurred secondary to

174 lung cancer ( $\mathrm{n}=1)$, hepatic failure (1), pulmonary embolism (1), renal failure (2), and sepsis (2). 
175

176

177

178

179

180

181

182

183

184

185

186

187

188

\section{Discussion}

The optimal management strategy for an infected lower extremity bypass graft or artery would be complete excision and in-line reconstruction with continuous autogenous vein. Unfortunately, availability of suitable vein having adequate caliber and length is lacking in many vascular patients with a previous history of bypass. ${ }^{6}$ In our cause, this was $96 \%$ of the population studied. As such, we routinely employ the use of CHs if the operation involves a potentially infected field. However, we do not routinely implant CHs for sterile-field bypasses given their dismal patency and limb salvage rates. ${ }^{7-10}$

CHs are harvested from multi-organ donors and preserved in dimethyl sulfoxide (DMSO) before being frozen in liquid nitrogen $\left(-196^{\circ} \mathrm{C}\right)$ for storage. ${ }^{11}$ Additionally, each $\mathrm{CH}$ vendor employs a unique preservation process to decrease antigenicity. The complex harvest and preservation process does incur a significant financial burden to the patient when this conduit is selected for bypass. ${ }^{12}$ When needed, grafts are thawed to room temperature and individually modified by the surgeon. After pressurization, the endothelial layer is slowly effaced and the tunica media infiltrated by leukocytes resulting in chronic fibrosis. ${ }^{13}$ This smoldering inflammatory response likely has a large role in late graft failure characterized by intense fibroplasia. ${ }^{14}$

mechanism. This effect has been postulated to be related to the presence of the conduit extracellular matrix allowing for the increased transfer of leukocytes and antibiotics into the perigraft space.15 Alternatively, it may be related to vendor-unique methods of tissue processing including the storage of grafts in the presence of antibiotics. ${ }^{16}$ 
We report one and 2-year patencies of $50 \%$ and $38 \%$ corresponding to limb salvage rates of $70 \%$ and $65 \%$. AFS during the same time periods were $58 \%$ and $52 \%$. Seven patients required amputation of the ischemic limb during the follow-up phase; however, amputation risk was frontloaded as all but one of the subjects lost their limb within 21 days of the index operation. This data clearly suggests a danger period for limb loss in the perioperative phase of infected-field repeat bypasses.

Surprisingly, robust contemporary series describing CH conduits in infected fields have been few and small. ${ }^{10,17}$ Brown et al. reviewed their experience with $\mathrm{CHs}$ in infected fields which included peripheral, but also, carotid and visceral non-aortic reconstructions. Their published experience described 39 total cases with a mean follow-up of 18 months. Mortality at 30-days was 2.6\%. Interestingly, graft reinfection did not occur in their population in contrast to our observed rate of $13 \%$. Unfortunately, their 1-year patency was not published. ${ }^{7}$ The largest series of cryopreserved vein bypasses was reported in 2003 of 240 consecutive cases in both clean and infected fields. The majority (89\%) were performed for rest pain or tissue loss. The percentage of infected limbs were not published. The authors did note an overall 30\% 1-year primary patency and $80 \%$ limb salvage for all comers. ${ }^{8}$

\footnotetext{
We found a high reintervention rate of $63 \%$ in our study. Twenty-eight additional reinterventions following the index procedure were documented in our 24 patients. The most common indication was for stenosis, occlusion, or necrotizing soft tissue requiring a combination of angioplasty, thrombectomy, redo bypass, and debridement. It seems apparent that the index bypass for this indication cannot be considered the final and definitive operation. Therefore, before selecting the patient for limb salvage or primary amputation, it is imperative to disclose the risk of prolonged hospitalization and additional interventions. Based on our experience, we have adopted
} 
225 the use of cryopreserved homografts in infected fields with concurrent placement of a muscle flap

226 when possible for the sole purpose of limb salvage. After clearance of the infection, consideration

227 should be made into reoperation with an alternative conduit to improve long-term outcomes.

228

Unfortunately, the retrospective nature of this study makes it impossible to be sure all

230 adverse events were tracked and captured. As many patients were referred to us from outside

231 vascular surgeons and hospital systems - their follow-up often occurred external to our records.

232 Regardless, the limited adverse events abstracted in this study illustrates well the poor prognosis of

233 this population. Another potential confounder present is inherent to a group practice, where

234 multiple vascular surgeons perform operations per their expertise, often on the same patient. Thus,

235 standard protocol and procedure were lacking. 


\section{Conclusion}

237

CHs are an accepted alternative to continuous autogenous vein for redo bypasses in the

239 setting of an infected field. However, the surgeon should be aware of the increased incidence of

240 amputation, death, and reintervention prior to offering $\mathrm{CH}$ limb salvage for this difficult population. 


\section{Disclosures}

242

243 The authors have no conflicts of interest to disclose. 
1. Mamode N, Scott RN. Graft type for femoro-popliteal bypass surgery. Cochrane Database of Systematic Reviews. 1999(2).

2. Kent KC, Whittemore AD, Mannick JA. Short-term and midterm results of an all-autogenous tissue policy for infrainguinal reconstruction. Journal of vascular surgery. 1989;9(1):107-114.

3. Taylor LM, Edwards JM, Brant B, Phinney ES, Porter JM. Papers of the North Pacific Surgical AssociationAutogenous reversed vein bypass for lower extremity ischemia in patients with absent or inadequate greater saphenous vein. The American Journal of Surgery. 1987;153(5):505-510.

4. Chaikof EL, Fillinger MF, Matsumura JS, Rutherford RB, White GH, Blankensteijn JD, et al. Identifying and grading factors that modify the outcome of endovascular aortic aneurysm repair. Journal of vascular surgery. 2002;35(5):1061-1066.

5. Hardman RL, Jazaeri O, Yi J, Smith M, Gupta R. Overview of Classification Systems in Peripheral Artery Disease. Seminars in Interventional Radiology. 2014;31(4):378-388.

6. Kreienberg PB, Darling Iii RC, Chang BB, Champagne BJ, Paty PSK, Roddy SP, et al. Early results of a prospective randomized trial of spliced vein versus polytetrafluoroethylene graft with a distal vein cuff for limb-threatening ischemia. Journal of vascular surgery. 2002;35(2):299-306.

7. Brown KE, Heyer K, Rodriguez H, Eskandari MK, Pearce WH, Morasch MD. Arterial reconstruction with cryopreserved human allografts in the setting of infection: A single-center experience with midterm follow-up. Journal of vascular surgery. 2009;49(3):660-666.

8. Farber A, Major K, Wagner WH, Cohen JL, Cossman DV, Lauterbach SR, et al. Cryopreserved saphenous vein allografts in infrainguinal revascularization: analysis of 240 grafts. Journal of vascular surgery. 2003;38(1):15-21.

9. Gentile AT, Lee RW, Moneta GL, Taylor Jr LM, Edwards JM, Porter JM. Results of bypass to the popliteal and tibial arteries with alternative sources of autogenous vein. Journal of vascular surgery. 1996;23(2):272-280.

10. Hartranft CA, Noland S, Kulwicki A, Holden CR, Hartranft T. Cryopreserved saphenous vein graft in infrainguinal bypass. Journal of vascular surgery. 2014;60(5):1291-1296.

11. Martin RS, Edwards WH, Mulherin JL, Edwards WH, Jenkins JM, Hoff SJ. Cryopreserved saphenous vein allografts for below-knee lower extremity revascularization. Annals of surgery. 1994;219(6):664-672.

12. Huber AJ, Brockbank K, Riemann I, Schleicher M, Schenke-Layland K, Fritze O, et al. Preclinical evaluation of ice-free cryopreserved arteries: structural integrity and hemocompatibility. Cells Tissues Organs. 2012(1422-6421 (Electronic)).

13. Calhoun A, Baur G, Porter J, Houghton D, Templeton JW. Fresh and cryopreserved venous allografts in genetically characterized dogs. J Surg Res. 1977(0022-4804 (Print)).

14. Carpenter JP, Tomaszewski JE. Human saphenous vein allograft bypass grafts: immune response. Journal of vascular surgery. 1998(0741-5214 (Print)).

15. Vogt PR, Brunner-LaRocca H-P, Lachat M, Ruef C, Turina MI. Technical details with the use of cryopreserved arterial allografts for aortic infection: Influence on early and midterm mortality. Journal of vascular surgery. 2002;35(1):80-86.

16. Camiade C, Goldschmidt P, Koskas F, Ricco J-B, Jarraya M, Gerota J, et al. Optimization of the Resistance of Arterial Allografts to Infection: Comparative Study with Synthetic Prostheses. Annals of vascular surgery. 2001;15(2):186-196.

17. Castier Y, Francis F, Cerceau P, Besnard M, Albertin J, Fouilhe L, et al. Cryopreserved arterial allograft reconstruction for peripheral graft infection. Journal of vascular surgery. 2005;41(1):3037. 


\section{Legends}

Table 1: Comorbidities

Table 2: Operative characteristics

Table 3: Culture results

Table 4: Peri-operative management and results

Table 5: Extended outcomes

Figure 1: Kaplan-Meier analysis for AFS over time for patients receiving cryopreserved homografts in an infected surgical field. The inputs displayed are all-cause mortality and major (above-ankle) amputation. 


\begin{tabular}{|l|l|}
\hline Comorbidity & Incidence \\
\hline HTN & $92 \%$ \\
\hline Active Smoker & $58 \%$ \\
\hline HLD & $54 \%$ \\
\hline CAD & $42 \%$ \\
\hline Obesity & $33 \%$ \\
\hline DM & $25 \%$ \\
\hline CRI (Cr > 1.5) & $21 \%$ \\
\hline CVD & $17 \%$ \\
\hline Arrhythmia & $17 \%$ \\
\hline HD & $13 \%$ \\
\hline COPD & $8 \%$ \\
\hline Rutherford's Ischemia Score & $4.3 \pm 0.6$ \\
\hline SVS Risk Score & $3.9 \pm 2.1$ \\
\hline
\end{tabular}




\begin{tabular}{|l|l|}
\hline Operative Characteristics & Incidence \\
\hline Emergent & $29 \%$ \\
\hline Septic & $8 \%$ \\
\hline Loss of Incorporation & $92 \%$ \\
\hline PSA & $33 \%$ \\
\hline Purulence & $46 \%$ \\
\hline Wound Culture Positive & $75 \%$ \\
\hline Muscle Flap & $46 \%$ \\
\hline
\end{tabular}




\begin{tabular}{|l|l|l|l|}
\hline Patient \# & Graft Material & Culture Results & ESBL/MRSA \\
\hline 1 & Synthetic & S. aureus, S. marascens & No \\
\hline 2 & Synthetic & Coagulase- Staph & No \\
\hline 3 & Synthetic & $\begin{array}{l}\text { Coagulase } \text { Staph, } \text { P. } \\
\text { aeruginosa, Citrobacter }\end{array}$ & No \\
\hline 4 & Synthetic & P. aeruginosa & No \\
\hline 5 & Synthetic & S. aureus & Yes \\
\hline 6 & Synthetic & Klebsiella & No \\
\hline 7 & Vein & P. aeruginosa & Yes \\
\hline 8 & Synthetic & S. aureus, Enterococcus & No \\
\hline 9 & Synthetic & Coagulase- Staphylococcus & No \\
\hline 10 & Synthetic & S. aureus & No \\
\hline 11 & Synthetic & Corynebacterium & No \\
\hline 12 & Native Artery & P. aeruginosa & No \\
\hline 13 & Synthetic & Corynebacterium & No \\
\hline 14 & Synthetic & S. aureus & Yes \\
\hline 15 & Synthetic & Coagulase- Staphylococcus & No \\
\hline 16 & Synthetic & Enterococcus & No \\
\hline 17 & Synthetic & Corynebacterium & No \\
\hline
\end{tabular}




\begin{tabular}{|l|l|}
\hline Post-Operative Regimen & Incidence \\
\hline Antiplatelets & $88 \%$ \\
\hline Anticoagulation & $38 \%$ \\
\hline Antibiotics & $100 \%$ \\
\hline Antibiotic Duration & $4.6 \pm 2.2$ Weeks \\
\hline 30-day Outcomes & Incidence \\
\hline MACE & $4 \%$ \\
\hline Major Amputation & $21 \%$ \\
\hline Mortality & $4 \%$ \\
\hline AFS & $75 \%$ \\
\hline Bleeding & $13 \%$ \\
\hline Reintervention & $38 \%$ \\
\hline
\end{tabular}




\begin{tabular}{|l|l|}
\hline Long-Term Outcomes & Incidence \\
\hline Follow-up & $27.9 \pm 20.4$ Months \\
\hline LOS & $14.8 \pm 16.3$ Days \\
\hline Primary Patency & $17.4 \pm 18.2$ Months \\
\hline 1-yr Primary Patency & $50 \%$ \\
\hline 1-yr AFS & $54 \%$ \\
\hline 2-yr Primary Patency & $38 \%$ \\
\hline 2-yr AFS & $52 \%$ \\
\hline Reintervention Rate & $63 \%$ \\
\hline Reinfection & $13 \%$ \\
\hline Bleeding & $13 \%$ \\
\hline Major Amputation & $29 \%$ \\
\hline Death & $54 \%$ \\
\hline
\end{tabular}




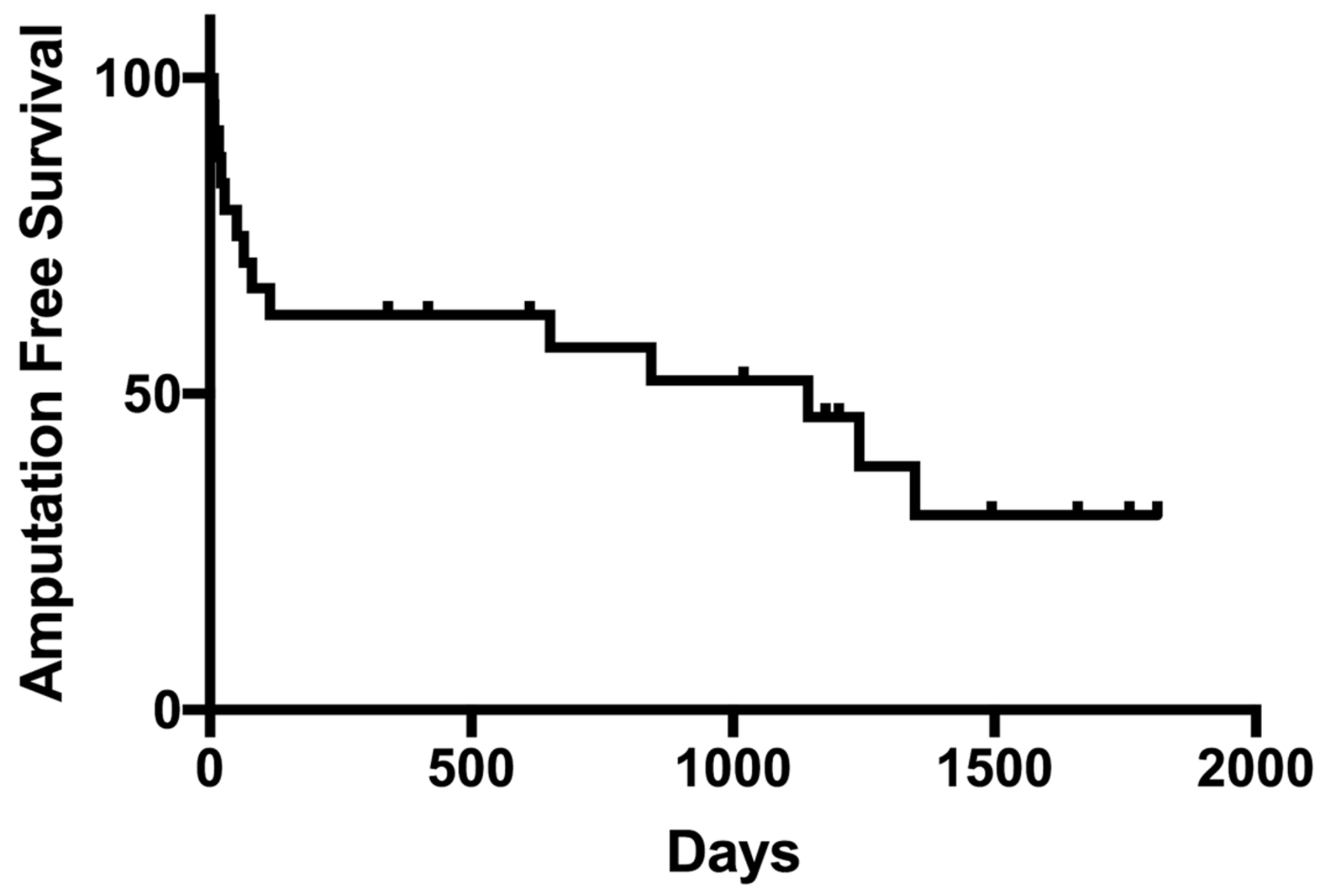

